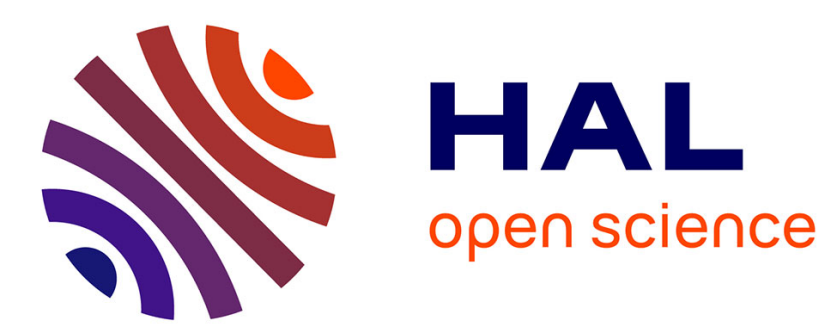

\title{
Fusome as a Cell-Cell Communication Channel of Drosophila Ovarian Cyst
}

\author{
Jean-René Huynh
}

\section{To cite this version:}

Jean-René Huynh. Fusome as a Cell-Cell Communication Channel of Drosophila Ovarian Cyst. F. Baluska, D. Volkmann \& P.W. Barlow. Cell-Cell Channels, Landes Biosciences, sous presse, 2005. hal-00013440

\section{HAL Id: hal-00013440 https://hal.science/hal-00013440}

Submitted on 8 Feb 2006

HAL is a multi-disciplinary open access archive for the deposit and dissemination of scientific research documents, whether they are published or not. The documents may come from teaching and research institutions in France or abroad, or from public or private research centers.
L'archive ouverte pluridisciplinaire HAL, est destinée au dépôt et à la diffusion de documents scientifiques de niveau recherche, publiés ou non, émanant des établissements d'enseignement et de recherche français ou étrangers, des laboratoires publics ou privés. 


\title{
CHAPTER
}

\section{Fusome as a Cell-Cell Communication Channel of Drosophila Ovarian Cyst}

\author{
Jean-René Huynh*
}

\begin{abstract}
I

$\mathrm{n}$ most animal species, female and male gametes are produced within clusters of germ cells which share a common cytoplasm through cell-cell channels. In Drosophila ovaries, these cells synchronise their divisions and specialise one cell of the cluster as the future egg. Both processes are organised by a germline-specific organelle of communication called the fusome. Untill recently, the fusome has remained largely mysterious despite a hundred years of research on its composition, formation and functions. Novel results have now suggested several molecular mechanisms to explain how the fusome synchronises the divisions by controlling cell-cycle regulators and how it determines and polarises the future egg by organising the microtubule cytoskeleton. Importantly, a structure similar to the fusome has been identified during Xenopus oogenesis, suggesting that it is widely conserved from invertebrates to vertebrates, and that it thus serves an essential function.
\end{abstract}

\section{Introduction}

In most animal species, male and female gametes start their differentiation as groups of germ cells with synchronous phases of development, indicating extensive communication between these cells. ${ }^{1}$ Accordingly, it was found that these cells (called "cystocytes") share a common cytoplasm through cytoplasmic bridges, thus forming a syncytium referred to as a cyst. This specific form of cell-cell channel originates from a series of incomplete cell divisions of a cyst-founder cell called a cystoblast. The bridges are derived from persistent mid-bodies in the arrested cleavage furrows. ${ }^{2,3}$ Male gametes retain these intercellular bridges during most of their differentiation. It has been suggested that they may serve not only to synchronise their development, but also to share gene products after the completion of meiosis, as half of the sperms will inherit one $\mathrm{X}$ chromosome and the other half a Y chromosome. ${ }^{4,5}$ Synchronisation and efficient sharing might thus alleviate the differences between advantaged and disadvantaged sperm. In females, intercellular bridges can be limited to the synchronous phase of divisions, as in mammalian ovaries, or they can form stable structures called ring canals as shown in Drosophila. ${ }^{1}$ In this fruitfly, communication between the germ cells has an additional function as it allows the specialization of one cell of the cluster as the oocyte (the future egg), while the remaining germ cells of the cyst become nurse cells, which provide the oocyte with nutrients and cytoplasmic components through the ring canals.

A second and equally important feature of Drosophila cyst development is the presence of a large cytoplasmic structure called the fusome, which links all the cells of the cluster through the ring canals. ${ }^{6}$ Although the behaviour of the fusome has been best studied in insects, ${ }^{7}$ a similar structure has been recently described in Xenopus laevis during the formation of female germline cysts. ${ }^{8}$ The fusome seems thus widely conserved from invertebrates to vertebrates, which suggests that it serves

*Jean-Rene Huynh, Institut Jacques-Monod, CNRS, Universités Paris 6 et 7, 2, place Jussieu, F-75251 Paris. Cedex 05, France. Email: huynh@ijm.jussieu.fr

Cell-Cell Channels, edited by Frantisek Baluska, Dieter Volkmann, and Peter W. Barlow. (C)2005 Eurekah.com. 
an important function. The fusome is mainly made of densely packed vesicles filling up the ring canals. It was thus first described as a "plug" within the ring canals and thought to block any communication between cells of the cyst. ${ }^{6}$ Surprisingly, it was found in Drosophila ovaries that in the absence of the fusome, cells of the same cluster divide asynchronously and fail to specify an oocyte, despite the presence of ring canals. ${ }^{9}, 10$ This demonstrates a key role of the fusome as a channel of communication between the cells for the synchronisation and differentiation of the germline cyst. In this review, I will mainly focus on the fusome of the Drosophila ovaries, where it has been possible to study its formation and functions at the genetic, cellular and molecular levels. Other aspects of Drosophila oogenesis have been summarized elsewhere, including ring canals formation (this issue and ${ }^{11,12}$ ), germline stem cells regulation ${ }^{13}$ and differentiation of the cyst. ${ }^{14,15}$

The Drosophila ovary is composed of 16-20 ovarioles, each of which contains a chain of progressively more and more mature egg chambers. ${ }^{16}$ Germline cysts are produced throughout the life of the adult fly, by the divisions of germline stem cells localised in a specialised region called the germarium situated at the anterior tip of each ovariole (Fig. 1). The germarium has been divided into 4 regions according to the developmental stage of the cyst. Oogenesis begins in region 1, when a germline stem cell (GSC) divides asymmetrically to produce a posterior cystoblast, and a new GSC, which remains attached to the neighbouring somatic cells at the anterior. ${ }^{13,17}$ The cystoblast then undergoes precisely four rounds of mitosis with incomplete cytokinesis to form a cyst of 16 germline cells, which are interconnected by ring canals. Once the 16 cell cyst has formed, it enters the region 2a of the germarium. At this stage, all the cells of one cyst look the same, but by the time it reaches region $2 \mathrm{~b}$, one cell will have differentiated as an oocyte. This differentiation can be followed with several types of marker summarized in Figure 1 . In region $2 \mathrm{~b}$, the cyst changes shape and becomes a one cell-thick disc that spans the whole width of the germarium. Oocyte-specific factors are now concentrated into the oocyte and a microtubule organising centre (MTOC) is clearly seen in this cell. At the same time, somatic follicle cells start to migrate and surround the cyst. As the cyst moves down to region 3 (also called stage 1), it rounds up to form a sphere with the oocyte always lying at the posterior pole. The cyst then leaves the germarium into the vitellarium.

\section{Formation of the Fusome}

The fusome was first described in 1886 by Platner as Verbindungsbrücken ("bridging connections") in the spermatocytes of several insects using iron hematoxylin staining and light microscopy. ${ }^{18}$ In 1901, Giardina recorded the origin and formation of the fusome in the female germline cyst of the diving beetle Dytiscus. ${ }^{2}$ His observations and conclusions remain still valid and turn out to be general principles of fusome formation. Between 1960 and 1970, King, Mahowald and colleagues described a region of the cytoplasm of the Drosophila cysts as rich in vesicles and fibrills using electron microscopy. ${ }^{19-21}$ This structure was then recognized as the fusome by Telfer in $1975 .{ }^{6}$ More recently Allan Spradling's lab, taking advantage of Drosophila new genetic tools, described the fusome at the molecular and cellular levels using fluorescent immunostaining and confocal microscopy., Finally, using transgenic flies expressing GFP-tagged markers, Mary Lilly's group was able to observe the fusome using live confocal microscopy and photobleaching techniques. ${ }^{26}$ After more than a century of observations and experiments, a highly dynamic picture of the formation of fusome has emerged.

\section{A Complex Choreography}

The fusome sensu stricto appears during the first division of the cystoblast. It arises from a similar structure present in the germline stem cells (GSC) called a spectrosome, which is also made of vesicles and whose molecular composition differs only slightly form that of the fusome. ${ }^{24}$ The first appearance of the spectrosome itself has been reported in the germ cells of the gastrulating embryo. ${ }^{27}$ In the adult, the spectrosome is stably anchored at the anterior side of the GSC, in contact with the adherens junctions between the GSC and the overlying cap cells (Figs. 1, 2). ${ }^{28}$ During the GSC division, one pole of the mitotic spindle is anchored by the spectrosome thus orientating the division along the antero-posterior axis of the germarium (Fig. 2). ${ }^{27,29}$ The cystoblast 


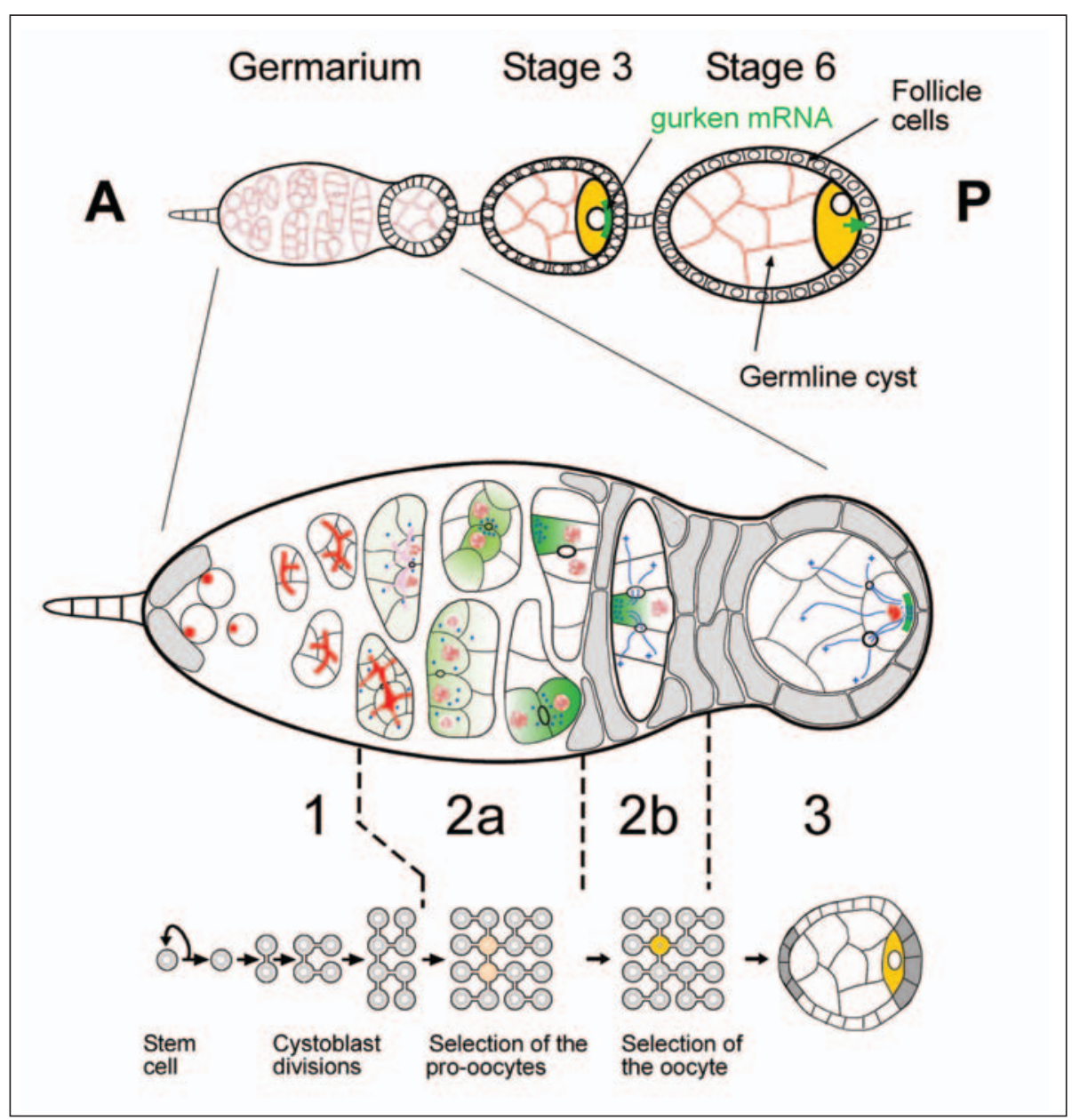

Figures. 1 Drosophila early oogenesis. Each ovariole is made of a chain of more and more mature egg chambers towards the posterior $(\mathrm{P})$. An egg chamber comprises 16 germline cells surrounded by a monolayer of follicle cells. The egg chambers are produced in a specialised structure, called the germarium, at the anterior (A) of the ovariole. The germarium is divided into 4 morphological regions along the antero-posterior axis. The germline stem cells reside at the anterior tip of the germarium in contact with the somatic cap cells (grey cells on the left) and divide to produce cytoblasts, which divide four more times in region 1 to produce 16 cell germline cysts that are connected by ring canals. The stem cells and cystoblasts contain a spectrosome (red circles), which develops into a branched structure called the fusome, which orients each division of the cyst. In early region $2 \mathrm{a}$, the synaptonemal complex (SC, red lines) forms along the chromosomes of the 2 cells with 4 ring canals (the pro-oocytes) as they enter meiosis. The SC then appears transiently in the 2 cells with 3 ring canals, before becoming restricted to the pro-oocytes in late region $2 \mathrm{a}$. By region $2 \mathrm{~b}$, the oocyte has been selected, and is the only cell to remain in meiosis. In region $2 \mathrm{a}$, cytoplasmic proteins, mRNAs and mitochondria (green), and the centrosomes (blue circles) progressively accumulate at the anterior of the oocyte. In region $2 \mathrm{~b}$, the minus-ends of the microtubules are focused in the oocyte, and the plus-ends extend through the ring canals into the nurse cells. The follicle cells (grey) also start to migrate and surround the germline cells. As the cyst moves down to region 3, the oocyte adheres strongly to the posterior follicle cells and repolarises along its antero-posterior axis, with the MT minus-ends and specific cytoplasmic components now localised at the posterior cortex. A color version of this figure is available online at www.Eurekah.com. 


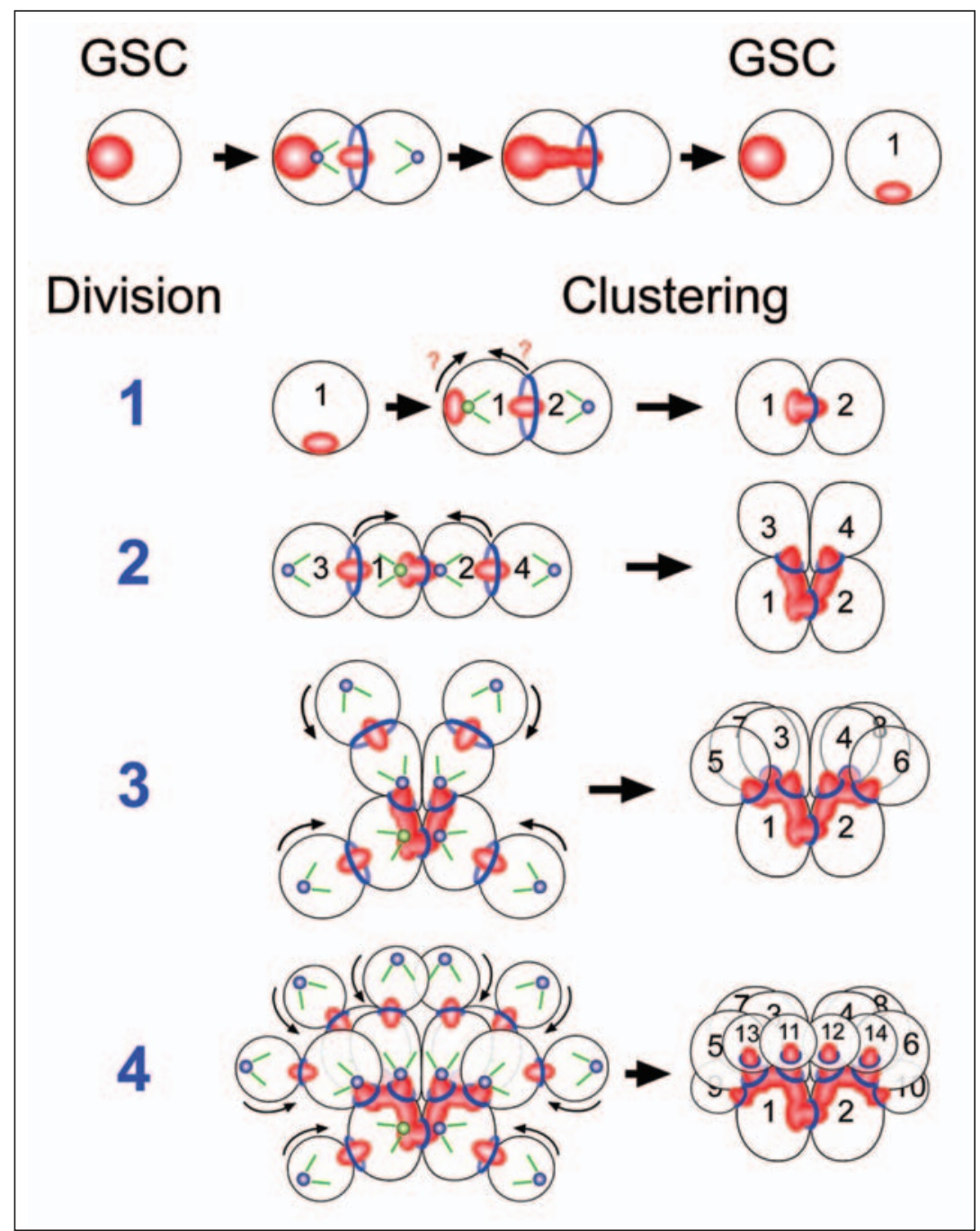

Figures 2. Formation of the fusome. The spectrosome (red) of the GSC anchors one pole of the mitotic spindle and orients the division along the anterior-posterior axis. A new fusome plug forms into the transient ring canal. The spectrosome elongates to fuse with the plug. The cytoplasmic bridge is then severed and one third of the spectrosome/fusome is inherited by the cystoblast while two-third remains in the GSC, marking this division as clearly asymmetric. The spectrosome/fusome (red) of the cystoblast (1) interacts with one of the centrosomes (green and blue spheres) to anchor one pole of the mitotic spindle (green lines), during the first incomplete division. A fusome plug (red) forms in the arrested furrow or ring canal (blue). The spectrosome (or "original" fusome) and the fusome plug come together to fuse. The direction of these movements is not known (?). The same mechanism is repeated for division 2, 3 and 4: (1) One pole of each mitotic spindle is anchored by the fusome. (2) A new fusome plug forms into each ring canal. (3) the ring canals move centripetally for the fusome plugs to fuse with the central fusome (black arrows). This behaviour has several crucial consequences: (1) cystocyte (1) has more fusome than the other cystocytes; (2) the same centrosome (green sphere) could be inherited by the cystocyte (1) from division 1 through division 4; and (3) the fusome always marks the anterior of cystocyte (1), after the clustering of the ring canals. A color version of this figure is available online at www.Eurekah.com. 
is then produced toward the posterior and the renewed GSC stays at the anterior of the germarium, in a specialized cellular environment called a "niche". ${ }^{13}$ At the end of telophase, a transient ring canal forms between the GSC and the cystoblast. New fusome material accumulates in this ring canal and the spectrosome elongates from the anterior side of the GSC to fuse with the new fusome. ${ }^{22,29}$ The cytoplasmic bridge is then severed and one third of the spectrosome/fusome is inherited by the cystoblast while two-third remains in the GSC, marking this division as clearly asymmetric. ${ }^{27,29}$ The spectrosome relocalises to the anterior side of the GSC, while the fusome takes a spherical shape at one end of the cystoblast.

The first mitosis of the cystoblast is very similar to the GSC division (Fig. 2). One pole of the mitotic spindle is anchored by the spherical fusome, a new fusome "plug" forms into the arrested furrow at the other end of the cell and comes to fuse with the "original" fusome. However, in contrast to the GSC division, cytokinesis is incomplete and both cells remain linked by a stable ring canal. Furthermore, although it is not known how the plug and the "original" fusome move to fuse together, the "original" fusome does not seem to elongate from one side of the cystoblast, as in the GSC. ${ }^{22}$ This division is also asymmetric as one cell contains the "original" fusome plus half of the plug, whereas the other cell only retains the other half of the fusome plug. At the next division, the two mitotic spindles again orient with one pole close to the fusome and new fusome plugs form in the two ring canals at opposite ends. ${ }^{22,30-32}$ The fusome plugs then move with their ring canal to fuse with the central fusome, which thus remains in the two previous cells. This asymmetric behaviour of the fusome is then repeated in the next two divisions (Figs. 2, 3). The oldest cell, therefore, retains the original fusome and accumulates four halves of fusome plugs. Thus, this cell has more fusome than all the other cells and can be identified throughout the divisions. The current model suggests that this cell will become the oocyte (see below). Once the 16 cells-cyst is formed, the fusome starts to break down and disappears. The behaviour of the Drosophila fusome reveals general morphogenetic principles of how germline cysts form and has important consequences on the polarisation of the female cyst. ${ }^{6,14}$

Firstly, a consequence of the synchronous divisions is that the number of cells per cyst is a power of two: $2^{\mathrm{n}}$, with $\mathrm{n}$ being the number of divisions. In Drosophila male and female $\mathrm{n}$ equals $4\left(2^{4}=16\right)$, but $\mathrm{n}$ can range from 1 to at least 8 (256 cells) in the Strepsipteran Elenchinus japonicus. ${ }^{14}$ The $2^{\mathrm{n}}$ rule seems also to apply to vertebrates as Xenopus cysts go through four synchronous divisions and contain 16 cells. $^{8}$

A second general principle is that germline cysts do not form linear clusters of cells but are organised along a "maximally branched" pattern of interconnections. It is a consequence of the anchoring of one pole of each mitotic spindle by the fusome. ${ }^{22,30-32}$ This orientation of the divisions ensures that one cell inherits all the previous interconnections (ring canals), while a new one is formed at the opposite end of the cell and thus branched off the central fusome. This orientation also leads to an invariant pattern of interconnections between the cells, with the two central or oldest cells having $\mathrm{n}$ ring canals, their daughter cells $\mathrm{n}-1$, etc. This pattern is important for the polarisation of the cyst as the oocyte always arises from one of the two cells with the greatest number (n) of ring canals. ${ }^{7}$

At each interphase, the fusome plugs and the associated ring canals move centripetally to fuse with the central fusome. This clustering of the ring canals at the centre of the cyst with the cells protruding outward creates a rosette-like structure (Fig. 2). This specific geometry is a third characteristic of the germline cysts. ${ }^{6,7}$ One function of the rosette shape could be to minimize the distances between cells inside the cyst and thus make cell-cell communication more efficient. ${ }^{14}$ This shape is later on stabilised by the formation of adherens junctions around the ring canals, as seen by the localisation of Armadillo, E-Cadherin and Bazooka and confirmed by EM sections (ref. 33 and A.P. Mahowald, personal communication).

Finally, the behaviour of the fusome reveals several levels of polarisation within the female cyst. As already mentioned, the invariant pattern of divisions restricts the oocyte fate to the two cells with the greatest number of ring canals. In addition, the centripetal movement of the ring canals along the cell membrane to fuse with the central fusome uncovers a polarity within each cell of the cyst 


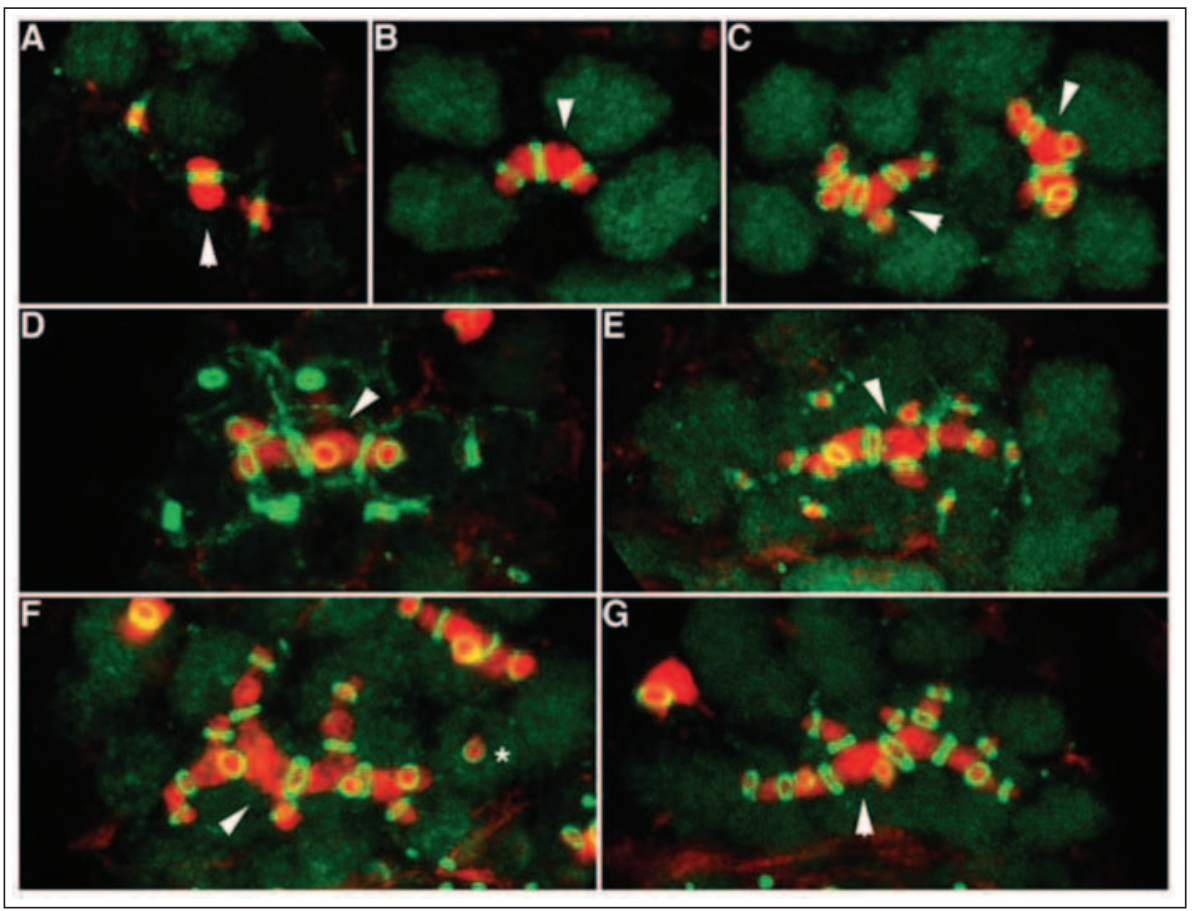

Figures 3. Growth of the fusome observed by confocal microscopy. Drosophila ovaries were stained with anti-anillin (ring canal, green) and anti-Hts (fusome, red) antibodies. Anterior is at the top in each panel. A) A 4-cell cyst in early-mid interphase. Two fusome plugs have formed, one in each new ring canal. B) A 4-cell cyst in late interphase. The plugs have fused with the original fusome, forming one continuous structure that spans the three ring canals. C) A pair of 8-cell cysts in late interphase. In each cyst, the four new plugs, in the four smallest ring canals, have already fused. D) A 16-cell cyst in early interphase. Fusomal material is beginning to accumulate in the new ring canals, but has not yet formed distinct plugs. Only seven of the eight new ring canals are visible here. E,F) 16-cell cysts in mid interphase. The plugs have enlarged and are beginning to fuse, though not all are fusing at the same rate. In E, only 14 ring canals are visible, the other is out of focus. In F, only one of the plugs (asterisk) has not yet fuse with the rest of the fusome. G) A 16-cell cyst in late interphase. All fusome plugs have fused; the fusome spans all 15 ring canals. One of the two cells with the most ring canals always contains more fusomal material than its sisters (arrowhead). Thus, throughout cyst formation, the fusome is asymmetrically distributed within the cyst. (Images courtesy of Allan Spradling, Department of Embryology, Carnegie Institution of Washington and Development, the Company of Biologists, UK, and reprinted with permission from: de Cuevas M, Spradling AC, Development 1998; 125:2781-2789.) ${ }^{22}$

(cystocytes). It is not known what drives the movement of the fusome plugs, but several evidences suggest that it could be microtubules based. Mutations in MT-binding proteins, Orbit/Mast and abnormal spindle (asp), or in an MT minus-end motor, dynein, and its associated regulator, Lis1, affect the formation of the fusome, which appears less branched. ${ }^{31,34-37}$ Consequently, mutant cysts are produced with less than 16 cells. It is not yet clear, however, if these mutations affect the movement and formation of the plugs, or the anchoring of the mitotic spindles to the fusome, or both. A direct role of the microtubules in fusome biogenesis has not yet been tested, using depolymerising drugs. The polarity of the plugs movement may be given by a difference between the two centrosomes. The centrosome anchored to the fusome may have the ability to attract the plug, in contrast to the opposite centrosome. Whatever the mechanism, it shows that the plugs are able to read a polarity within each cell. 
Furthermore, it is reasonable to assume that as early as the two-cell stage, the central fusome occupies a fix position, while it is the plugs which move toward it. The fusome therefore marks one side of each cell, which is at the center of the cyst (the "central" surface of each cell). In particular, in region $2 \mathrm{~b}$, the fusome remnant will always be located at the anterior of the oocyte, where all the cytoplasmic components accumulate to form a Balbiani body. ${ }^{38}$ Therefore, as early as the two-cell stage, the "central" localisation of the fusome marks the future anterior side of the oocyte. An anterior-posterior axis in the oocyte is thus inherited from the polarised cyst divisions. It is tempting to try to go one step earlier and to relate this polarity to the cystoblast division or even to the GSC division. However, there is no fix referential in the cystoblast, and for the moment it is not possible to tell if the "original" fusome is always at the posterior of the cystoblast, or if after the first cyst division, it is the plug, or the "original" fusome, or both, which move to fuse. ${ }^{22,29}$ Live imaging of these processes should help to solve these questions.

\section{Fusome Composition}

\section{The Fusome Is Made of ER-Derived Vesicles}

In 1901, Giardina described the fusome as a fibrillar material accumulated inside the arrested cleavage furrow and named it residue fusoriale to indicate that it was a remnant of the mitotic spindle. ${ }^{2}$ However, light microscopy could not allow the nature of the fibrillar material to be determined. A real breakthrough came when King, Mahowald and colleagues used electronic microscopy (EM) to describe the Drosophila germarium. ${ }^{19-21}$ They found an unusual organelle made of vesicles linking all the cells of the cyst through their ring canals (Fig. 4). This organelle excluded other components of the cytoplasm such as mitochondria and microtubules. This structure was then recognised by Telfer in 1975 as the fusome described earlier by Giardina and Hirschler. ${ }^{2,39}$ The next step was then to identify the nature of these vesicles and it remained a mystery until very recently. Mary Lilly and colleagues made transgenic flies expressing GFP-tagged proteins targeted to specific cellular subcompartments such as the Golgi and the endoplasmic reticulum (ER). ${ }^{26}$ They found that only the proteins targeted to the ER (Lys-GFP-KDEL, a lumenal ER protein and Sec61 $\alpha$-GFP an ER membrane protein) colocalised with the fusome, indicating that the fusome tubules are most similar to ER vesicles (Fig. 5). In an elegant experiment, they further showed that the fusome is continuous across all the cells of the cyst and is not made of independent vesicles packed together. They used Fluorescence Recovery after Photobleaching (FRAP) of Lys-GFP-KDEL and Sec61 $\alpha$-GFP to calculate their diffusion coefficient (Deff) within the fusome. They reasoned that if the fusome is made of discrete vesicles, both proteins should diffuse with the same speed, because their movements would be limited by the diffusion of the whole vesicle. In contrast, if the fusome is made of one continuous network of interconnected tubules, the lumenal protein Lys-GFP-KDEL should diffuse faster than Sec61 $\alpha$-GFP embedded in the more viscous ER membrane. They found the later case to be true and concluded that the fusome is continuous across the cyst. Moreover, they could also deplete the fluorescence of both proteins inside the fusome by repeatedly photobleaching the cytoplasmic ER of one cell of the cyst. This technique called FLIP (Fluorescence Loss in Photobleaching) demonstrates that the fusome is also interconnected with the cytoplasmic ER of each cell, which means that all the cells within a single cyst could share a common ER. These results are of primary importance as they could explain how the fusome synchronizes the development of the cells within the cyst by acting as a direct channel of communication. Interestingly, they further found that the continuity of the fusome is lost when the development of the cyst becomes asynchronous in region 2 of the germarium. ${ }^{26}$

However, we are still far from understanding how these ER-derived vesicles can form a plug inside a ring canal and then fuse to make a fusome. The final step of normal cytokinesis requires the insertion of new membranes at the cleavage furrow to separate the newly formed cells. Vesicles are transported to a compact structure rich in microtubules called the midbody, where they fuse with the existing plasma membrane. ${ }^{40}$ Interestingly, the fusome plugs are also formed and associated with the microtubules at the midbody during early interphase after each cyst division. ${ }^{3}$ It is thus possible that the plug is made of vesicles which are normally transported to the midbody but which, for an 


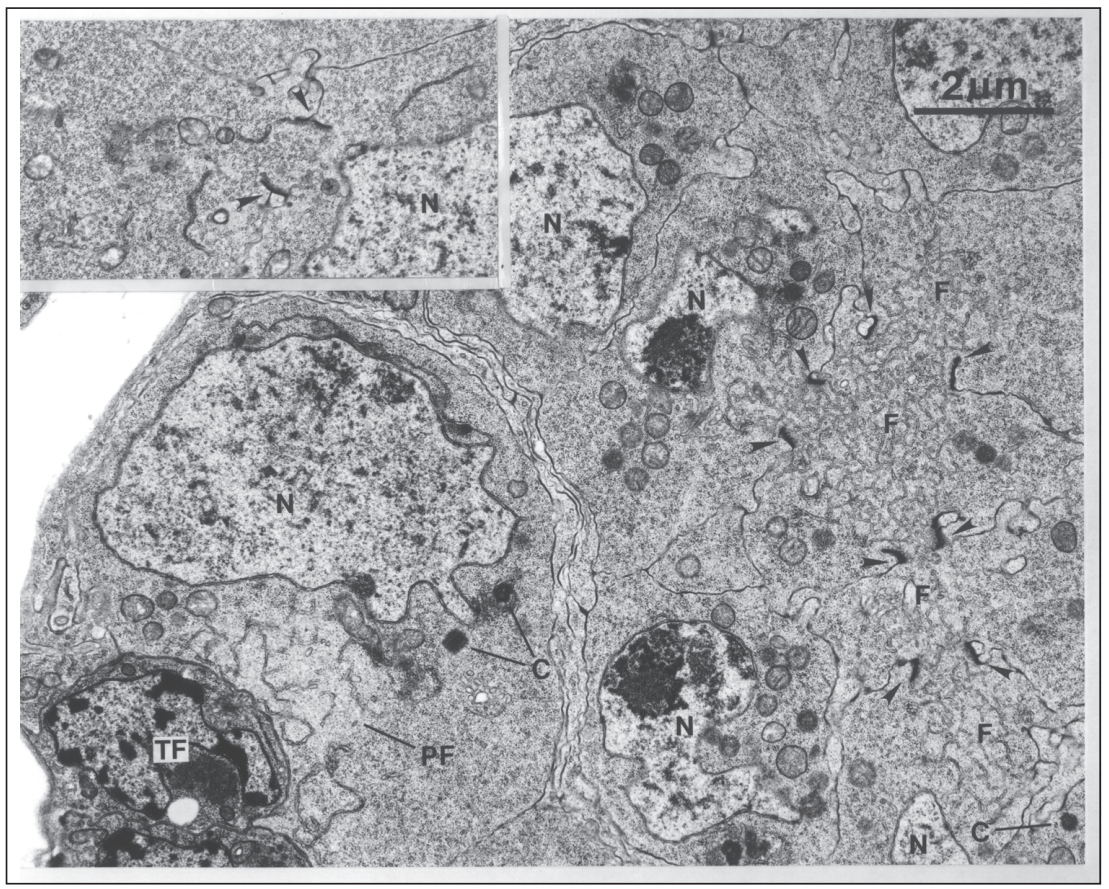

Figures 4. Electronic microscopy of the fusome and spectrosome in wild type and mutant germarium. Thin sections of the anterior region of the germarium from wild type and $h t$ mutant (inset) females shown at the same magnification. Anterior is to the left and posterior to the right. In the wild type germarium, a germline stem cell (GSC) with a large nucleus (N) lies closed to the somatic terminal filament cells (TF) at the anterior. The spectrosome (PF) is the region rich in vesicles between the GSC nucleus and the TF. A wild type cyst contains a large fusome (F) that extends through four ring canals (labeled by four pair of arrows) and associated with a centriole $(\mathrm{C})$. The fusome can be seen to contain small membranous vesicles and to exclude most ribosomes and mitochondria. In hts mutant female, these vesicles are absent (inset). The ring canal pictured appears to lack fusomal material entirely. (Image courtesy of Allan Spradling, Department of Embryology, Carnegie Institution of Washington and Development, the Company of Biologists, UK, and reprinted with permission from: Lin H, Yue L, Spradling A, Development 1994; 120:947-956. $)^{24}$

unknown reason, do not fuse with the plasma membrane. This could be due to the intrinsic nature of these vesicles or because they are coated with specific proteins.

\section{Molecular Composition of the Fusome}

The first hint about the molecular composition of the fusome came from the genetic study of the hts (hu-li-tai shao) gene in Drosophila. ${ }^{10,24}$ hts mutant flies produce germline cysts with a number of cells not following the $2^{\mathrm{n}}$ rule and which usually lack an oocyte and are only made of nurse cells. EM analysis of hts mutant germarium revealed a complete lack of fusome vesicles, further indicating that Hts was a good candidate as a fusome component (Fig. 4). The molecular cloning of Hts identified it as the Drosophila homologue of adducin, an actin and spectrin-binding protein constituting part of the membrane cytoskeleton. ${ }^{10}$ The membrane cytoskeleton is made of $\alpha$ and $\beta$-spectrin dimers linked together into a meshwork by junctional complexes composed of adducin, actin, protein 4.1 and tropomyosin. This lattice is attached to the plasma membrane by ankyrin and glycophorin. In erythrocytes, where it has been best studied, the sprectrin-based cytoskeleton was shown to modulate the mechanical properties of the plasma membrane. ${ }^{41}$ In addition, these membrane skeletal proteins are also associated with internal organelles such as the 


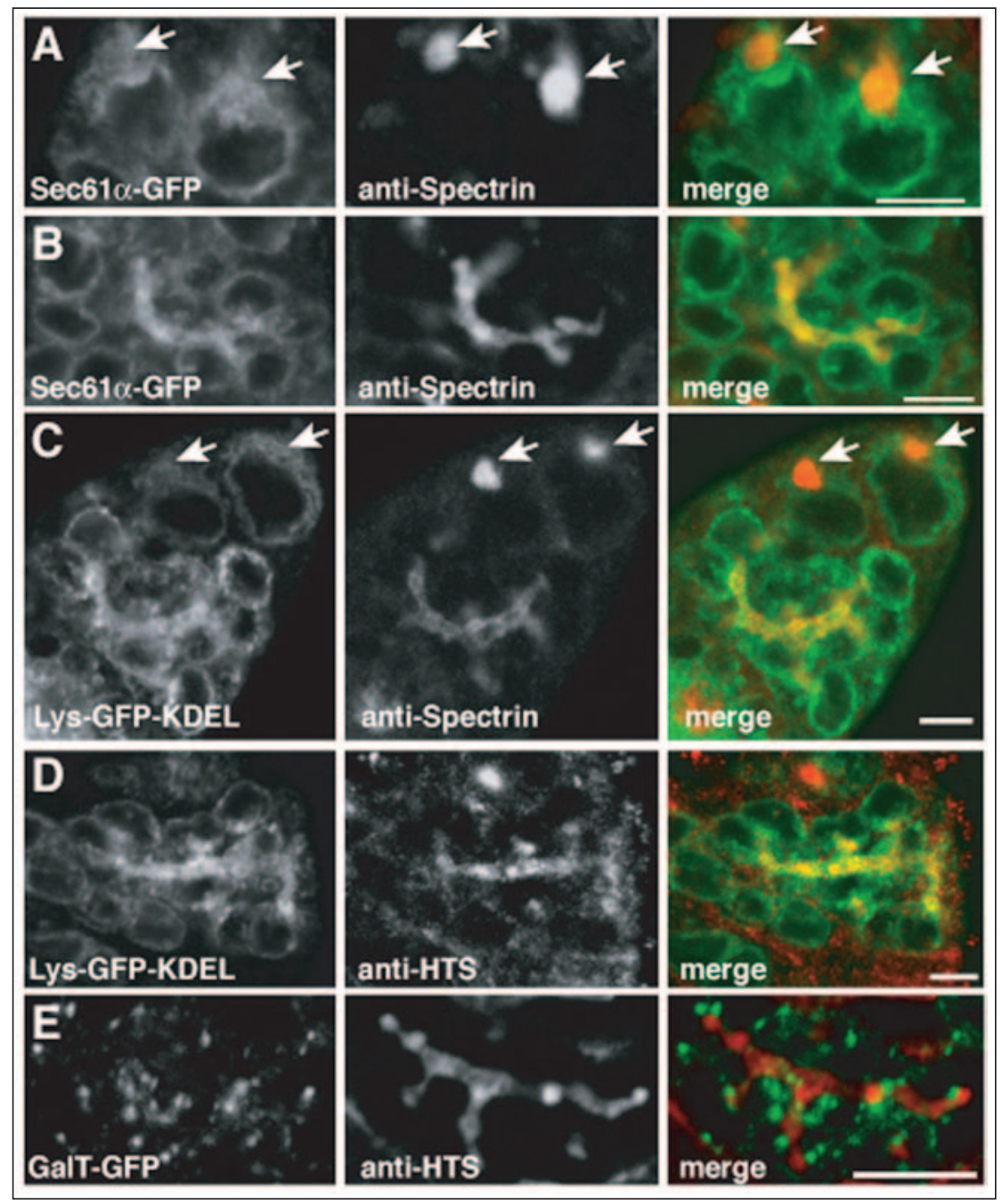

Figures 5. ER markers colocalise with the fusome. A-D) Germaria expressing the ER markers Sec61 $\alpha$-GFP $(\mathrm{A}, \mathrm{B})$ or Lys-GFP-KDEL (C,D) were fixed and labeled with the fusome markers anti-spectrin (A-C) or anti-Hts (D,E). Regions of colocalisation in the merged images (yellow) appear within the ER accumulations between cystocytes, but not within the cystocyte cytoplasm to reveal the ER origin of fusome membranes. In A and C, large spectrin accumulations (spectrosomes) in stem cells are indicated by white arrows. These structure do contain ER-labeling, though the structure are not substantially enriched with ER accumulations. E) A cyst from a GalTase-GFP (Golgi marker) ovary stained with anti-Hts. No significant colocalization between these markers was observed. Bars, 5 m. (Image courtesy of Mary Lilly, Cell Biology and Metabolism branch, NIH and the American Society for Cell Biology and reprinted with permission from: Snapp EL, Iida T, Frescas D et al, Mol Biol Cell 2004; 15:4512-4521. ${ }^{26}$ ) A color version of this figure is available online at www.Eurekah.com.

Golgi complex and the ER. ${ }^{42}$ Accordingly, $\alpha$ and $\beta$-spectrin were found to be part of the spectrosome and fusome, while ankyrin was mostly found in the spectrosome, suggesting that the molecular nature of the spectrosome/fusome is developmentally regulated (Fig. 3). ${ }^{9,24}$ In contrast, Drosophila homologues of protein 4.1 such as Coracle, Moesin or Merlin were not seen on the 
fusome. ${ }^{9,43} \mathrm{~F}$-actin is not detected reliably on the fusome in Drosophila female ovaries, whereas it is a major component of the male fusome. ${ }^{5}$ Strong fixation protocols seem to be required to detect F-actin on the female fusome, suggesting that F-actin is unstable. ${ }^{44}$ Furthermore, it has recently been shown that mutations in Scr64, Tec29 and kelch, which are involved in crosslinking actin filaments, affect the branching and maintenance of the fusome. ${ }^{44}$ Scr64 and Tec29 are tyrosine kinases which can phosphorylate Kelch and inhibit its actin-bundling ability. It has thus been proposed that the branching defects in Scr64, Tec29 and kelch mutants could be due to a failure to loosen actin filaments crosslinking around the fusome plugs to allow their fusion with the central fusome. Whatever the mechanism, these results at least suggest that the actin cytoskeleton could play an important role in the formation of the fusome.

In hts mutant most of the vesicles are gone, while Hts itself is only faintly detected on fusome remnants of $\alpha$-spectrin mutant cysts. ${ }^{9,24}$ Although it suggests a direct role of the membrane skeletal proteins in the biogenesis of the fusome, their precise function remains unknown. They could be involved in the formation of the fusome by coating some ER-vesicles to give them a specific identity and/or affinity. This would create a separate subdomain of the ER allowing the fusion of these vesicles and the formation of a distinct continuous structure. Alternatively, they could be required for the maintenance of the fusome by surrounding it with a rigid cytoskeleton, prohibiting its fusion with the plasma membrane and the dispersal of the vesicles.

Bam and its associated protein TER94 constitute a second class of components of the fusome. Bam is a novel protein, which localises both to the fusome of all germ cells and to the cytoplasm of dividing cysts. ${ }^{45,46}$ bam mutant cysts are mostly stopped at the one-cell cystoblast stage and contain a greatly reduced fusome, leading to the suggestion that Bam could be involved in the recruitment of vesicles to the fusome. Mutations in the benign gonial cell neoplasm (bgcn) gene induce an almost identical phenotype and interestingly, bgcn cysts also fail to localise Bam protein on the fusome, while $\mathrm{Hts}$ and $\alpha$-spectrin are normally localised. ${ }^{47}$ These results suggest that Bam localisation on the fusome is required for its function.

TER94 was found to directly interact with Bam in a two-hybrid screen and also to localise to the fusome. ${ }^{48}$ TER94 is a fly orthologue of the vertebrate TER proteins, required for vesicles fusion during the biogenesis of the ER and Golgi. This conserved function of TER94 supports the view that the fusome is formed by the fusion of ER-derived vesicles. However, TER94 function during the formation of the fusome could not be assessed as the existing alleles are cell lethal. Furthermore, as the bam mutant cysts do not progress beyond the one-cell stage, it remains unclear whether they contain a spectrosome or a fusome. One major difference between the fusome and the spectrosome is that the spectrosome is less dense in vesicles, which is similar to what has been described for the "reduced fusome" of bam mutant cysts. ${ }^{24,45}$ It is thus not clear if Bam is directly required for the formation of the fusome, or if the defects seen in bam mutant cysts are due to an early arrest of the cystoblast development.

A third class of fusome components is made of microtubules-associated proteins including the plus-end binding proteins, Orbit/Mast and CLIP-190, the MT minus-end motor, dynein, and the Drosophila homologue of Spectraplakin, shot. Orbit/Mast shows a cell-cycle dependent localisation on the fusome. ${ }^{36}$ During mitosis, it localises on the mitotic spindles and then on the spindle remnants at anaphase. During interphase, it is incorporated into the newly formed ring canals, the fusome plugs and then into the fusome. Dynein has been reported to localise on the fusome only during mitosis, however a clear colocalisation with known fusome components has not been demonstrated yet. ${ }^{31}$ Furthermore, as shown previously (see II.1), mutations in the dynein and Orbit/ Mast genes lead to the formation of fragmented and unbranched fusomes indicating that both proteins and probably the microtubule cytoskeleton are required for the formation of the fusome.

Finally, Shot and several other proteins have been reported to associate at least transiently with the fusome such as Cyclin A, P-Cyclin E, Cul1, 19S-S1 and Par-1, LKB1 (Par-4), Par-5. ${ }^{49-54}$ However, these proteins seem to be required for the differentiation of the germline cyst rather than the formation of the fusome per se. Their function will thus be discussed in this context below. 
Fusome Functions in the Formation and Differentiation of the Germline Cyst

In the absence of a fusome such as in $h t s$ and $\alpha$-spectrin mutant cysts, the formation and differentiation of the germline cyst is greatly affected. ${ }^{9,10}$ These mutant cysts contain a variable number of cells, which are usually less than 16 and not a factor of $2^{\mathrm{n}}$. In addition, they frequently lack an oocyte and are only made of nurse cells. These defects arise despite the presence of ring canals, indicating that the fusome is likely to be the main channel of cell-cell communication for the control of the cyst divisions and the differentiation of the oocyte during normal development. I will review recent exciting results which shed new light on the role of the fusome in both of these processes.

\section{The Fusome and the Regulation of the Cell-Cycle}

Drosophila germline cysts are produced by four rounds of synchronous divisions with incomplete cytokinesis. This requires at least two levels of regulation: one mechanism synchronising the cell cycle of each cystocyte and a second mechanism counting the number of division to precisely four. Direct and reverse genetic approaches have been used to find proteins involved in both mechanisms. The main cell-cycle regulators, Cyclins, were obvious candidates.

In Drosophila, CyclinA (CycA) associates with the cyclin-dependent kinase, $\mathrm{cdk} 1$, to promote the transition from G2 phase to mitosis. Interestingly, CycA was shown to localise on the fusome precisely during late G2 and early prophase, while it is degraded after metaphase (Fig. 6). ${ }^{55}$ The

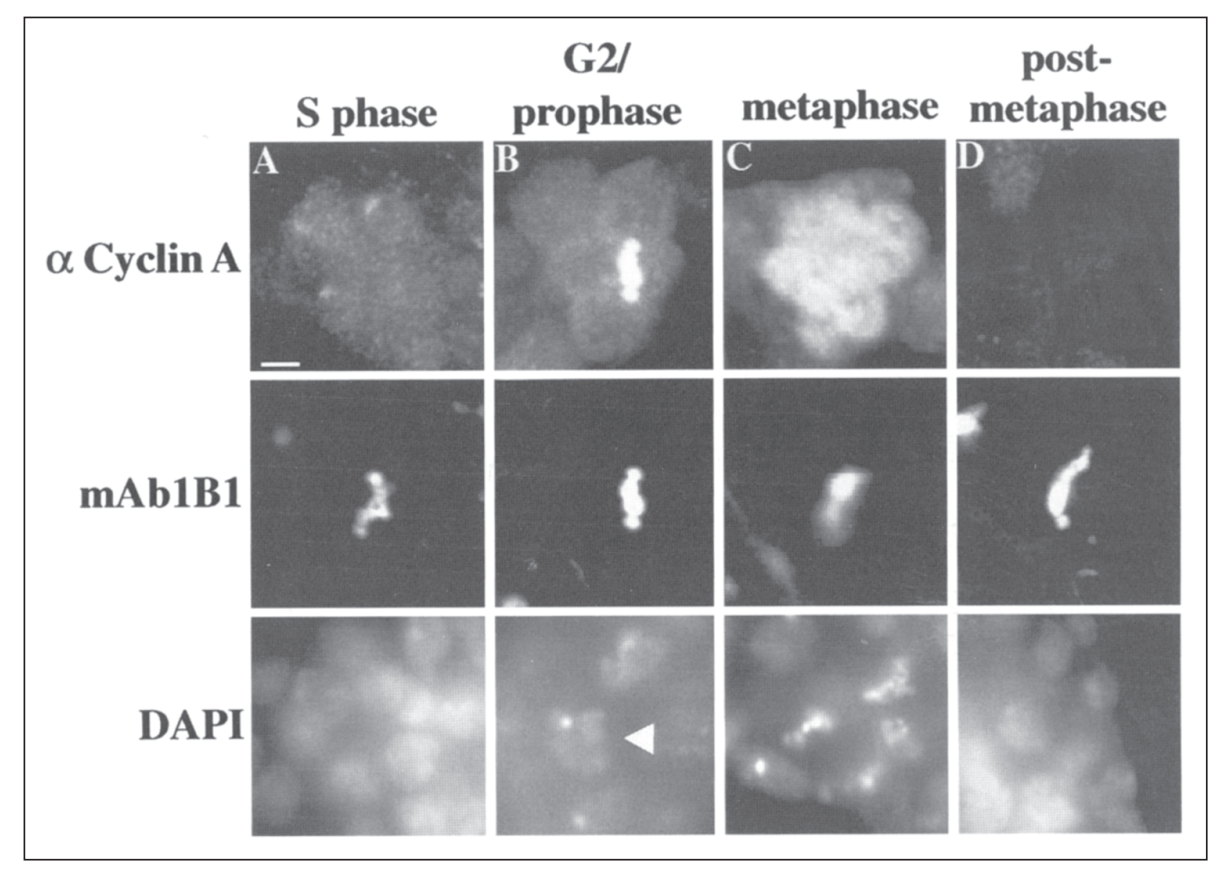

Figures 6. Cyclin A localisation on the fusome during the cystocyte divisions. (A-D) Ovaries were stained with antibodies raised against Drosophila $\mathrm{CycA}$ and the monoclonal antibody mAb1B1 which marks the fusome. DNA is labeled with DAPI. A single cyst in the indicated cell cycle stage is shown in each part. The DNA begins to condense at the G2/prophase transition (arrowhead). CycA precisely localises on the fusome at the G2/prophase transition and starts to be degraded at metaphase. For all images, not all cells within the dividing cyst are in the plane of focus. Bars, $5 \mu \mathrm{m}$. (Image courtesy of Mary Lilly, Cell Biology and Metabolism branch, NIH and the Academic Press, and reprinted from: Lilly MA, de Cuevas M, Spradling AC, Dev Biol 218:53-63, @2000, with permission from Elsevier. ${ }^{55}$ ) 
association of $\mathrm{CycA}$ with the whole fusome in late $\mathrm{G} 2$ phase could provide a mechanism to synchronise the entry of all cystocytes into mitosis. However, it remains unclear how the fusome could spatially equalize the activation of the CycA-cdk1 in all cells. One possibility is that phosphatases and kinases required for the activation of CycA-cdk1 could also be localised on the fusome. The activation of CycA-cdk1 at one point of the fusome could then feedback to the kinases and phosphatases and spread to all the cells by using in some way the fusome as a physical support. As mentioned earlier, in late G2 the fusome is continuous across the cyst and connect all the cells. ${ }^{26}$ The propagation of the active state of CycA-cdk1 would then synchronise the entry into mitosis.

The degradation of the cyclins appears equally important for the counting mechanism. Mutations in the encore gene induce one extra-division of the cyst and the formation of egg chambers containing 32 cells and one oocyte with five ring canals. ${ }^{56}$ Levels of CycA and Cyclin E, a G1 to S phase promoting cyclin, were shown to be abnormally high and to persist longer in region 2 of encore mutant germarium than in wild type, suggesting that an excess of activated cyclins could cause the extra division. ${ }^{53}$ Accordingly, the direct overexpression of $\mathrm{CycA}$ and $\mathrm{CycE}$ with a heat inducible promoter also induces the formation of 32-cells cysts, albeit at a low frequency. ${ }^{53,55,57}$ In addition, mutations affecting the degradation machinery also lead to high levels of CycA and CycE and greatly enhance the frequency on the extra round of mitosis in encore mutant ovaries. The intriguing twist of the story is that several members of the ubiquitin-dependent degradation pathway such as Cul1 and the proteasome $19 \mathrm{~S}$ subunit $\mathrm{S} 1$, as well as phosphorylated forms of CyclinE (P-cyclinE) marked for degradation, localise on the fusome. ${ }^{53}$ It was further shown that Encore physically interacts with the proteasome and CyclinE and was required for their localisation to the fusome, although Encore biochemical function remains unknown. Thus, the model proposes that Encore recruits the proteasome to the fusome to degrade simultaneously CyclinE in all cells, which would end the four rounds of mitosis. ${ }^{53}$ However, these results and the model do not explain why the cyst stops dividing after precisely four cycles. This question is central for developmental biologists, because many other processes such as cellularization of the Drosophila embryo and differentiation of the peripheral nervous system are also triggered after a precise number of divisions. How cells count the number of mitosis is a fascinating mystery and the divisions of the Drosophila cyst provides a powerful model to address it.

\section{The Fusome and the Determination of the Oocyte}

In Drosophila ovaries, as soon as the four divisions are complete and a 16-cells cyst is formed, the development of each cell of the cyst becomes asynchronous and an oocyte starts to differentiate. The determination of the oocyte has puzzled biologists for more than a century, because it arises from a syncytium of 16 sister cells that share the same cytoplasm. Several steps are required for one cell to become the female gamete (Fig. 1). Firstly, the oocyte needs to be selected from 16 sister cells in region 1 of the germarium. Secondly, the oocyte has to differentiate by accumulating oocyte-specific proteins and mRNAs in region 2 . Finally, the oocyte needs to be correctly polarised to maintain its identity in region 3. Although the fusome is only present in region 1 of the germarium, recent data suggest that it may well be required at several steps for the correct determination of the oocyte (Fig. 7).

\section{The Fusome and the Selection of the Oocyte}

Two main models have been proposed to explain how the oocyte is selected. One model is based on the symmetrical behaviour of the two pro-oocytes until mid-late region $2 \mathrm{a}$, and proposes that there is a competition between the two pro-oocytes to become the oocyte. ${ }^{58,59}$ The "winning" cell would become the oocyte, while the "losing" cell would revert to the nurse cell fate. However, the factor that could control this decision has remained elusive. A second model suggests that the choice of the oocyte is biased by the establishment of some asymmetry as early as the first cystoblast division, which is maintained until the overt differentiation of the oocyte. ${ }^{30,60}$ As described earlier, the formation of the fusome provides the strongest evidence in support of the second model. ${ }^{22,29-31,61}$ Indeed, the asymmetric inheritance of the "original fusome" during the cyst divisions could play the role of such an asymmetric cue. This model is further supported by analogy with the diving beetle 


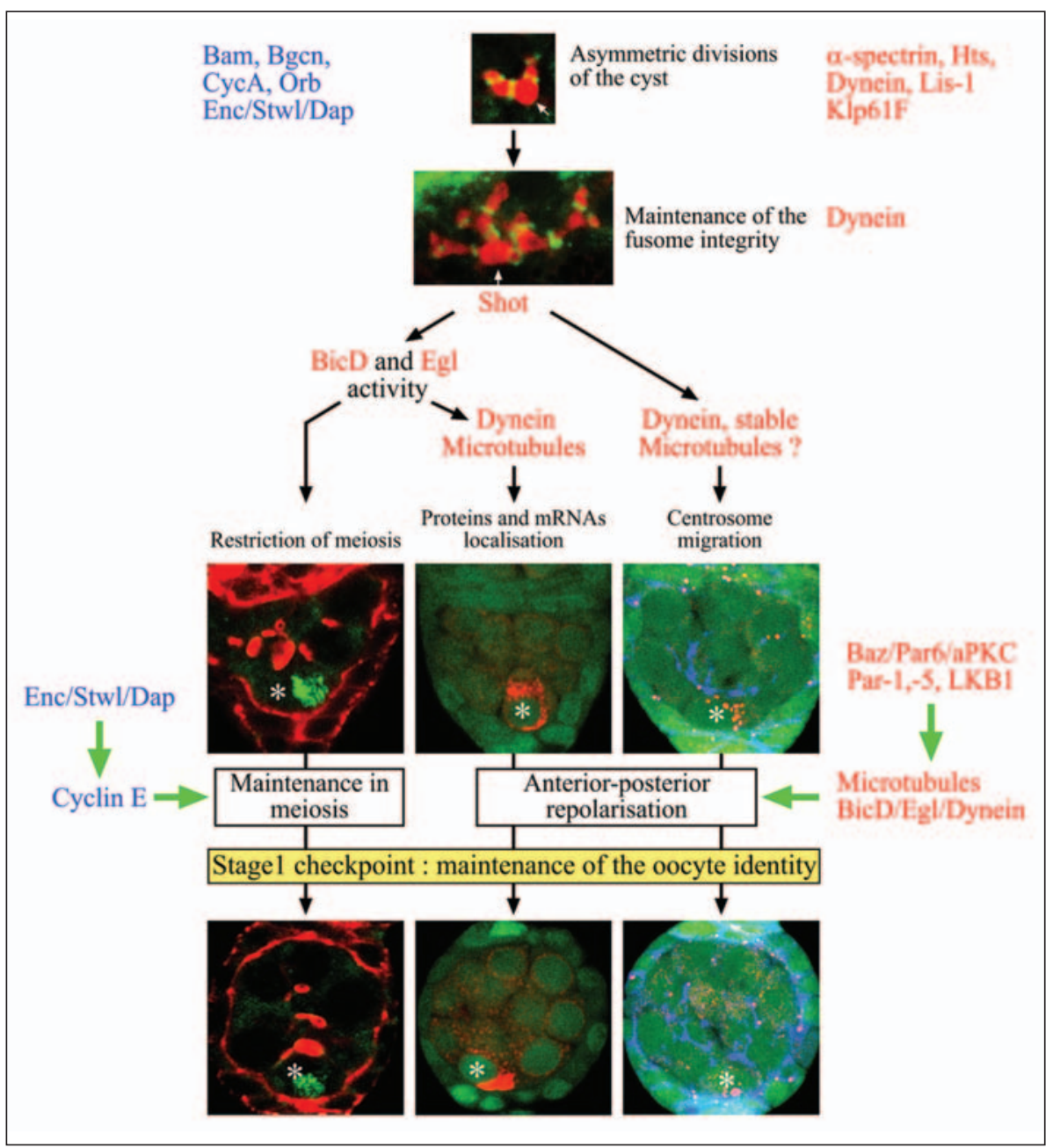

Figures 7. The early steps in the determination and polarisation of the Drosophila oocyte. The early differentiation of the oocyte is a multistep process. Genes involved at each step are indicated on the figure. The lists are not exhaustive. Actors of the cytoplasmic differentiation are in red, whereas regulators of the cell cycle are in blue. The top panel shows a 4-cell cyst ( $\alpha$-spectrin, in red, marks the fusome and anilin, in green, marks the ring canals). One cell has more fusome than the other cells (arrow). The panel below shows a 16-cell cyst after the last division with one cell having more fusome (arrow). There are three different pathways to restrict the oocyte identity to one cell (asterik). The left panel shows the actin in red and the synaptonemal complex in green. In the middle panels, a nuclear GFP is in green, and Orb (an oocyte-specific cytoplasmic protein) is in red. On the right panels, the $\gamma$-tubulin marks the centrosomes in red, the $\alpha$-spectrin marks the fusome in blue, and a nuclear GFP is in green. Orb and the centrosomes are clearly seen migrating from the anterior of the oocyte to the posterior, revealing the repolarisation of the oocyte. (Reprinted from: Huynh JR, St Johnston D, Curr Biol 14:R438-449, (C2004, with permission from Elsevier. ${ }^{15}$ ) A color version of this figure is available online at www.Eurekah.com.

Dytiscus, in which oogenesis is very similar to Drosophila. ${ }^{2,6}$ A Dytiscus cyst is formed of 15 nurse cells and one oocyte, resulting from four incomplete and synchronous divisions of a cystoblast. However, unlike Drosophila, the oocyte can be distinguished as early as the 2-cell stage because it 
contains a large ring of highly amplified rDNA. Moreover, the cell that inherits that ring of rDNA, also inherits the fusome. This also suggests that the early selection of the oocyte could be a general feature among insects. ${ }^{3,22}$ Unfortunately, in Drosophila most of the fusome has already degenerated by the time oocyte-specific proteins such BicD or Orb accumulate in a single cell in late region 2a. However, the preferential accumulation of the centrosomes and $o s k$, and orb mRNAs in one cell can be detected earlier in region $2 \mathrm{a}$, and this is always the cell with the most fusome. ${ }^{3,38}$ This is particularly obvious in egl and BicD mutants, in which the fusome perdures longer, and where the centrosomes clearly accumulate in the cell with the largest piece of fusome remnant. ${ }^{34}$ These data strongly suggest that the "original" fusome marks the future oocyte, in support of the second model. It does not rule out the possibility that both pro-oocytes can become the oocyte, but shows that if there is a competition, it is strongly biased.

What is the link between the asymmetric inheritance of the fusome and the selection of the oocyte? The simplest model is that an oocyte determinant is asymmetrically distributed at each division with the "original" fusome into the future oocyte. It has been proposed that one of the cystoblast centrioles could stay in contact with the fusome during each division, and because of the semi-conservative replication of the centrosome, could be inherited by the oocyte (Green centrosome in Fig. 2) ${ }^{60}$ Consequently, oocyte determinants could cosegregate with this centriole. Such a mechanism has been shown to mediate the segregation of $d p p$ and eve mRNAs into specific cells during the asymmetric divisions of the early Ilyanassa obsoleta embryo. ${ }^{62}$ Alternatively, the oocyte could inherit more of some protein or activity associated with the fusome, and this early bias could initiate a feedback loop that induces the transport of oocyte determinants towards this cell.

\section{The Fusome and the Differentiation of the Oocyte}

Even though strong evidence suggest that the oocyte is selected early in region 1, its identity only becomes obvious two days later, in late region 2a. The cyst differentiation in region $2 \mathrm{a}$ is gradual and two-fold. In the cytoplasm, the oocyte accumulates specific components and organelles, and in the nucleus, it enters meiosis and arrests in prophase I. Recent results have uncovered a complex picture in which multiple processes function in parallel to restrict different types of oocyte-specific components to one cell (for a detailed information see our recent review). ${ }^{15}$ However, all of these probably depend on the initial polarity of the fusome, which may act in three distinct ways for the differentiation of the oocyte.

Firstly, the fusome organises a polarised network of dynamic microtubules (MTs) that direct the localisation of oocyte-specific proteins and mRNAs into one cell. Indeed, one of the main features of cyst differentiation is the formation of a microtubule array that is polarised towards the oocyte and extends through the ring canals into the other cells of the cyst. ${ }^{63}$ Since the MT form along the fusome, this polarisation has been suggested to be a direct read out of the fusome polarity. ${ }^{3}$ MTs are essential for the determination of the oocyte, since treatments with the MT depolymerising drug, colchicine, result in the failure to localise the oocyte-specific proteins or mRNAs asymmetrically within these cysts and in the formation of egg chambers with 16 nurse cells and no oocyte. ${ }^{64}$ Furthermore, mutations in the adaptor proteins Bicaudal-D (Bic-D), egalitarian (egl) and the dynein light chain produce a very similar phenotype, suggesting that the transport of these proteins and mRNAs is MT-dependent and uses the dynein-dependent transport of cargoes that are linked to the motor through $\mathrm{BicD}, \mathrm{Egl}$ and the dynein light chain. ${ }^{31,65-67}$ Secondly, the fusome also nucleates stable MTs that are associated with the Drosophila spectroplakin, Shot. Indeed, colchicine disrupts the localisation of proteins and mRNAs to the oocyte, but not the migration of the centrioles or the restriction of meiosis to one cell. ${ }^{34,68}$ Since MT-destabilising drugs like colchicine only affect dynamic MTs, one possibility is that the centrioles migrate along stable MTs that are not affected by these treatments. In support of this view, it has recently been found that antibodies against acetylated tubulin, a marker for stable MTs, label a population of

MTs associated with the fusome. ${ }^{54}$ Furthermore, mutations in the Drosophila spectroplakin, Shot disrupt these stable microtubules without affecting the fusome itself, and this blocks the migration of the centrioles, as well as mRNAs and proteins, into one cell. Shot is a component of the 
fusome, and contains a GAS domain, which has been shown to bind and stabilise MTs. This suggests a model in which Shot assembles stable MTs on the fusome, along which the centrioles migrate. The situation is less clear for the restriction of meiosis, as it is only partially disrupted in shot germline clones. This raises the possibility that a third MT-independent pathway reads the fusome polarity directly to control the entry into meiosis. ${ }^{34,68}$

In summary, the current model suggests that although the fusome starts to degenerate in early region $2 \mathrm{a}$, it acts as a matrix to organise the restriction of oocyte-specific proteins, centrioles and meiosis to a single cell by at least three distinct pathways (Fig. 7). However, what molecular mechanisms regulate the different pathways and how these pathways interpret the fusome polarity remains unknown.

\section{The Fusome and the Early Polarisation of the Oocyte}

When a germline cyst reaches region $2 \mathrm{~b}$, the oocyte specific proteins and mRNAs, as well as the centrosomes and mitochondria have been transported along the fusome into the presumptive oocyte. These components remain associated with the fusome remnants and therefore accumulate at the anterior of the oocyte to form a Balbiani body. ${ }^{38}$ When the oocyte moves through region 3, all of the components of the Balbiani body disassociate and move around the oocyte nucleus to form a tight crescent at the posterior cortex. This movement is the first sign of anterior-posterior polarity in the oocyte, and is a crucial step in the maintenance of its identity. ${ }^{51,69}$

The importance of this polarisation step was first demonstrated by the analysis of mutations affecting members of a conserved group of genes called the par genes, which specifically disrupt this process (reviewed in ref. 70). The Drosophila par genes, which include par-1, par-3 (bazooka), LKB1 (par-4), par-5 (14-3-3), par-6 and $a P K C$, were first discovered in C. elegans where they are required for the polarisation of the one-cell zygote. They were later shown to be widely required from $C$. elegans to Drosophila and Human for the polarisation of many cell types. ${ }^{71}$ In Drosophila, egg chambers mutant for any of the par genes contains 16 nurse cells and lack an oocyte. The oocyte appears to be selected normally, as the centrosomes, meiotic markers, and Orb accumulate in one cell in region $2 \mathrm{~b} / 3 .^{51}$ These components do not translocate to the posterior of the oocyte in region 3 , however, and the oocyte de-differentiates as a nurse cell, by exiting meiosis and becoming polyploid. Intriguingly, Par-1, LKB1 (Par-4) and Par-5 localise on the fusome, while Par-3 and aPKC form rings of adherens junctions around the ring canal. ${ }^{33,49,51,52}$ However, none of these genes seem to be required for the formation of the fusome itself, nor during the cyst divisions. What could be the link between the fusome and the early polarisation of the oocyte? One possibility is that the par genes are required with the fusome to polarize each cell of the cysts during its divisions in region 1 . This polarisation of the oocyte would then organise its cytoskeleton required for the translocation of the Balbiani body to the posterior pole in region 3. As already mentioned, the fusome marks the "central" surface of each cell and could thus serve to asymmetrically localised the PAR proteins and polarise each cell of the cyst, including the future oocyte. Alternatively, as the Balbiani body forms around the fusome remnants, the localisation of the PAR proteins on the fusome and its remnants could be required directly for the MT-dependent transport of the Balbiani vesicles to the posterior of the oocyte. Although mainly unexplored, the links between the fusome, the par genes and the early polarisation of the oocyte are promising.

\section{Conclusions and Perspectives}

Although I have focused on Drosophila oogenesis in this review, the role of the fusome as a channel of communication is likely to be widely conserved. Indeed, groups of germ cells dividing synchronously have been reported in females and males of most if not all animal species. Given the primary role of the fusome in synchronising these cells, it suggests that a fusome could exist in those same species at least during the divisions. In support of this view, a structure similar to the Drosophila fusome at the morphological and molecular levels has been recently found in Xenopus. ${ }^{8}$ Indeed, Xenopus germline cysts are formed by four rounds of incomplete mitosis and contain a spectrin-rich structure branching across the cyst (Fig. 8). However, in Xenopus all the germ cells become oocyte. 

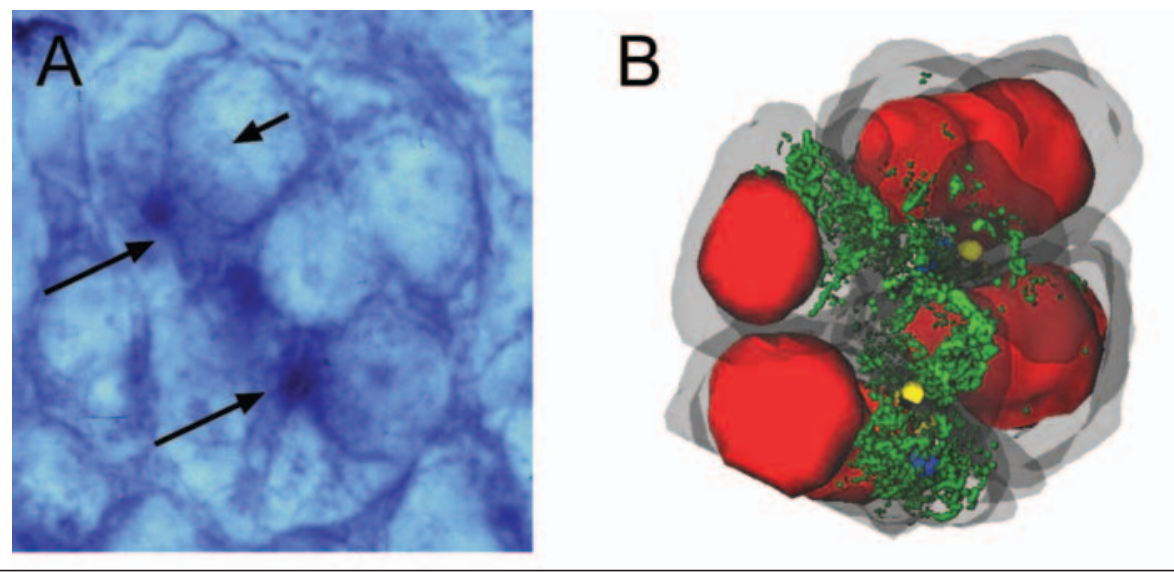

Figures 8. Fusome of Xenopus laevis female ovaries. A) Xenopus 8-cell cyst stained with an antibody raised against spectrin. Long arrows point to spectrin in the cytoplasm where the primary mitochondrial clouds (PMC) and the fusome localise. Short arrow indicates the nucleus. B) Three-dimensional reconstruction of an 8-cell cyst. Cytoplasm is grey, nuclei are red, mitochondria of PMC are green, centrioles blue and ring canals are yellow. PMC, ring canals and centrioles face each other and are located centripetally in the "rosette" conformation. This reconstruction was made from 38 serial ultrathin sections. (Images courtesy of Malgorzata Kloc and Laurence Etkin, Department of Molecular Genetics, University of Texas, and reprinted from: Kloc M, Bilinski S, Dougherty MT et al, Dev Biol 266:43-61, (C2004 with permission from Elsevier. ${ }^{8}$ )

This suggests that the role of the fusome in the selection and differentiation of the oocyte may be a secondary function restricted to species which differentiate accessory germ cells such as the nurse cells in Drosophila.

We are only starting to understand the origin, formation and functions of the fusome and many questions remain unanswered. However, rapid progresses have been made recently thanks to the use of powerful genetic tools available in Drosophila. Indeed, several genes required for the formation of the fusome and proteins associated with the fusome have been found in large scale genetic screens and reverse genetic approaches. In addition, it is now possible to study the fusome in vivo using confocal live imaging and transgenic flies expressing specific fluorescently tagged proteins. ${ }^{26}$ Furthermore, the recent success in culturing GSCs in a cell medium opens exciting new possibilities to study the differentiation of the germline cyst in vitro. ${ }^{72}$ The early steps of Drosophila oogenesis thus provide a useful paradigm to comprehend the role of the fusome and therefore to better understand the formation of functional gametes crucial to the sexual reproduction.

\section{Acknowledgments}

I thank Jean-Antoine Lepesant for critical reading of the manuscript. I also thank Laurence Etkin, Malgorzata Kloc, Mary Lilly and Allan Spradling for the images illustrating this review. I am supported by the CNRS and a grant from Association pour la Recherche sur le Cancer (A.R.C.).

\section{References}

1. Pepling ME, de Cuevas M, Spradling AC. Germline cysts: A conserved phase of germ cell development. Trends Cell Biol 1999; 9:257-262.

2. Giardina A. Origine dell'oocite e delle cellule nutrici nel Dytiscus. Int Mschr Anat Physiol 1901; $18: 417-484$

3. Grieder N, De Cuevas M, Spradling A. The fusome organizes the microtubules network during oocyte differentiation in Drosophila. Development 2000; 127:4253-4264.

4. Braun RE, Behringer RR, Peschon JJ et al. Genetically haploid spermatids are phenotypically diploid. Nature 1989; 337:373-376. 
5. Hime G, Brill J, Fuller M. Assembly of ring canals in the male germ line from structural components of the contractile ring. J Cell Sci 1996; 109:2779-2788.

6. Telfer W. Development and physiology of the oocyte-nurse cell syncytium. Adv Insect Physiol 1975; 11:223-319.

7. Buning J. The Insect Ovary: Ultrastructure, previtellogenic growth and evolution. New-York: Chapman and Hall, 1994.

8. Kloc M, Bilinski S, Dougherty MT et al. Formation, architecture and polarity of female germline cyst in Xenopus. Dev Biol 2004; 266:43-61.

9. de Cuevas M, Lee JL, Spradling AC. $\alpha$-spectrin is required for germline cell division and differentiation in the Drosophila ovary. Development 1996; 124:3959-3968.

10. Yue L, Spradling A. hu-li tai shao, a gene required for ring canal formation during Drosophila oogenesis, encodes a homolog of adducin. Genes Dev 1992; 6:2443-2454.

11. Cooley L. Drosophila ring canal growth requires Src and Tec kinases. Cell 1998; 93:913-915.

12. Hudson AM, Cooley L. Understanding the function of actin-binding proteins through genetic analysis of Drosophila oogenesis. Annu Rev Genet 2002; 36:455-488.

13. Spradling A, Drummond-Barbosa D, Kai T. Stem cells find their niche. Nature 2001; 414:98-104.

14. de Cuevas M, Lilly MA, Spradling AC. Germline cyst formation in Drosophila. Annu Rev Genet 1997; 31:405-428.

15. Huynh JR, St Johnston D. The origin of asymmetry: Early polarisation of the Drosophila germline cyst and oocyte. Curr Biol 2004; 14:R438-449.

16. Spradling A. Developmental genetics of oogenesis. In: Bate M, Martinez-Arias A, eds. The development of Drosophila melanogaster. New-York: Cold Spring Harbor Laboratory Press, 1993:1-70.

17. Gonzalez-Reyes A. Stem cells, niches and cadherins: A view from Drosophila. J Cell Sci 2003; 116:949-954.

18. Platner G. Die Karyokinese bie den Lepidopteran als Grundlage fur eine Theorie der Zellteilung. Int Mschr Anat Physiol 1886; 3:341-398.

19. Koch E, King R. The origin and early differentiation of the egg chamber of Drosophila. J Morph 1966; 119:283-304.

20. Mahowald AP. The formation of ring canals by cell furrows in Drosophila. Z Zellforsch Mikrosk Anat 1971; 118:162-167.

21. Mahowald AP. Ultrastructural observations on oogenesis in Drosophila. J Morphol 1972; 137:29-48.

22. de Cuevas M, Spradling AC. Morphogenesis of the Drosophila fusome and its implications for oocyte specification. Development 1998; 125:2781-2789.

23. Lin H, Spradling A. Fusome asymmetry and oocyte determination in Drosophila. Dev Genet 1995; 16:6-12.

24. Lin H, Yue L, Spradling A. The Drosophila fusome, a germline-specific organelle, contains membrane skeletal proteins and functions in cyst formation. Development 1994; 120:947-956.

25. Zaccai M, Lipshitz H. Role of Adducin-like (hu-li tai shao) mRNA and protein localization in regulating cytoskeletal structure and function during Drosophila oogenesis and early embryogenesis. Dev Genet 1996; 19:249-257.

26. Snapp EL, Iida T, Frescas D et al. The fusome mediates intercellular endoplasmic reticulum connectivity in Drosophila ovarian cysts. Mol Biol Cell 2004; 15:4512-4521.

27. Lin H, Spradling A. A novel group of pumilio mutations affects the asymmetric division of germline stem cells in the Drosophila ovary. Development 1997; 124:2463-2476.

28. Xie TAS. A niche maintaining germline stem cells in the Drosophila ovary. Science 2000; 290:328-330.

29. Deng W, Lin H. Spectrosomes and fusomes anchor mitotic spindles during asymmetric germ cell divisions and facilitate the formation of a polarized microtubule array for oocyte specification in Drosophila. Dev Biol 1997; 189:79-94.

30. Lin H, Spradling AC. Fusome asymmetry and oocyte determination in Drosophila. Dev Gen 1995; 16:6-12.

31. McGrail M, Hays TS. The microtubule motor cytoplasmic dynein is required for spindle orientation during germline cell divisions and oocyte differentiation in Drosophila. Development 1997; 124:2409-2419.

32. Storto PD, King RC. The role of polyfusomes in generating branched chains of cystocytes during Drosophila oogenesis. Dev Genet 1989; 10:70-86.

33. Huynh JR, Petronczki M, Knoblich JA et al. Bazooka and PAR-6 are required with PAR-1 for the maintenance of oocyte fate in Drosophila. Curr Biol 2001; 11:901-906.

34. Bolivar J, Huynh JR, Lopez-Schier H et al. Centrosome migration into the Drosophila oocyte is independent of $\mathrm{BicD}$ and egl, and of the organisation of the microtubule cytoskeleton. Development 2001; 128:1889-1897. 
35. Liu Z, Xie T, Steward R. Lis1, the Drosophila homolog of a human lissencephaly disease gene, is required for germline cell division and oocyte differentiation. Development 1999; 126:4477-4488.

36. Mathe E, Inoue $\mathrm{YH}$, Palframan W et al. Orbit/Mast, the CLASP orthologue of Drosophila, is required for asymmetric stem cell and cystocyte divisions and development of the polarised microtubule network that interconnects oocyte and nurse cells during oogenesis. Development 2003; 130:901-915.

37. Riparbelli MG, Massarelli C, Robbins LG et al. The abnormal spindle protein is required for germ cell mitosis and oocyte differentiation during Drosophila oogenesis. Exp Cell Res 2004; 298:96-106.

38. Cox RT, Spradling AC. A Balbiani body and the fusome mediate mitochondrial inheritance during Drosophila oogenesis. Development 2003; 130:1579-1590.

39. Hirschler J. Gesetzmassigkeiten in den Ei-Nahrzellenverbanden. Zool Jb Abt Allg Zool Physiol $1945 ; 61: 141-236$.

40. Straight AF, Field CM. Microtubules, membranes and cytokinesis. Curr Biol 2000; 10:R760-770.

41. Bennett V, Baines AJ. Spectrin and ankyrin-based pathways: Metazoan inventions for integrating cells into tissues. Physiol Rev 2001; 81:1353-1392.

42. Devarajan P, Stabach PR, Mann AS et al. Identification of a small cytoplasmic ankyrin (AnkG119) in the kidney and muscle that binds beta I sigma spectrin and associates with the Golgi apparatus. J Cell Biol 1996; 133:819-830.

43. McCartney BM, Fehon RG. Distinct cellular and subcellular patterns of expression imply distinct functions for the Drosophila homologues of moesin and the neurofibromatosis 2 tumor suppressor, merlin. J Cell Biol 1996; 133:843-852.

44. Djagaeva I, Doronkin S, Beckendorf SK. Src64 is involved in fusome development and karyosome formation during Drosophila oogenesis. Dev Biol 2005.

45. McKearin D, Ohlstein B. A role for the Drosophila bag-of-marbles protein in the differentiation of cystoblasts from germline stem cells. Development 1995; 121:2937-2947.

46. McKearin D, Spradling A. bag-of-marbles: A Drosophila gene required to initiate both male and female gametogenesis. Genes Dev 1990; 4:2242-2251.

47. Lavoie CA, Ohlstein B, McKearin DM. Localization and function of Bam protein require the benign gonial cell neoplasm gene product. Dev Biol 1999; 212:405-413.

48. Leon A, McKearin D. Identification of TER94, an AAA ATPase protein, as a Bam-dependent component of the Drosophila fusome. Mol Biol Cell 1999; 10:3825-3834.

49. Benton R, Palacios IM, St Johnston D. Drosophila 14-3-3/PAR-5 is an essential mediator of PAR-1 function in axis formation. Dev Cell 2002; 3:659-671.

50. Cox DN, Lu B, Sun T et al. Drosophila par-1 is required for oocyte differentiation and microtubule organization. Curr Biol 2001; 11:75-87.

51. Huynh JR, Shulman JM, Benton R et al. PAR-1 is required for the maintenance of oocyte fate in Drosophila. Development 2001; 128:1201-1209.

52. Martin SG, St Johnston D. A role for Drosophila LKB1 in anterior-posterior axis formation and epithelial polarity. Nature 2003; 421:379-384.

53. Ohlmeyer JT, Schupbach T. Encore facilitates SCF-Ubiquitin-proteasome-dependent proteolysis during Drosophila oogenesis. Development 2003; 130:6339-6349.

54. Roper K, Brown NH. A spectraplakin is enriched on the fusome and organizes microtubules during oocyte specification in Drosophila. Curr Biol 2004; 14:99-110.

55. Lilly MA, de Cuevas M, Spradling AC. Cyclin A associates with the fusome during germline cyst formation in the Drosophila ovary. Dev Biol 2000; 218:53-63.

56. Hawkins N, Thorpe J, Schupbach T. Encore, a gene required for the regulation of germ line mitosis and oocyte differentiation during Drosophila oogenesis. Development 1996; 122:281-290.

57. Lilly M, Spradling A. The Drosophila endocycle is controlled by Cyclin E and lacks a checkpoint ensuring S-phase completion. Genes Dev 1996; 10:2514-2526.

58. Carpenter A. Electron microscopy of meiosis in Drosophila melanogaster females. I Structure, arrengement, and temporal change of the synaptonemal complex in wild-type. Chromosoma 1975; 51:157-182.

59. Carpenter A. Egalitarian and the choice of cell fates in Drosophila melanogaster oogenesis. Ciba Found Symp 1994; 182:223-246, (246-254).

60. Theurkauf WE. Microtubules and cytoplasm organisation during Drosophila oogenesis. Dev Biol 1994; 165:352-360.

61. Storto P, King R. The role of polyfusomes in generating branched chains of cystocytes during Drosophila oogenesis. Dev Genet 1989; 10:70-86.

62. Lambert JD, Nagy LM. Asymmetric inheritance of centrosomally localized mRNAs during embryonic cleavages. Nature 2002; 420:682-686. 
63. Theurkauf W, Alberts M, Jan Y et al. A central role for microtubules in the differentiation of Drosophila oocytes. Development 1993; 118:1169-1180.

64. Koch E, Spitzer R. Multiple effects of colchicine on oogenesis in Drosophila; induced sterility and switch of potencial oocyte to nurse-cell developmental pathway. Cell Tissue Res 1983; 228:21-32.

65. Mach JM, Lehmann R. An Egalitarian-BicaudalD complex is essential for oocyte specification and axis determination in Drosophila. Genes Dev 1997; 11:423-435.

66. Navarro C, Puthalakath H, Adams JM et al. Egalitarian binds dynein light chain to establish oocyte polarity and maintain oocyte fate. Nat Cell Biol 2004; 6:427-435.

67. Ran B, Bopp R, Suter B. Null alleles reveal novel requirements for Bic-D during Drosophila oogenesis and zygotic development. Development 1994; 120:1233-1242.

68. Huynh JR, St Johnston D. The role of BicD, Egl, Orb and the microtubules in the restriction of meiosis to the Drosophila oocyte. Development 2000; 127:2785-2794.

69. Paré C, Suter B. Subcellular localization of Bic-D:GFP is linked to an asymmetric oocyte nucleus. J Cell Sci 2000; 113:2119-2127.

70. Pellettieri J, Seydoux G. Anterior-posterior polarity in C. elegans and Drosophila -PARallels and differences. Science 2002; 298:1946-1950.

71. Ohno S. Intercellular junctions and cellular polarity: The PAR-aPKC complex, a conserved core cassette playing fundamental roles in cell polarity. Curr Opin Cell Biol 2001; 13:641-648.

72. Kai T, Williams D, Spradling AC. The expression profile of purified Drosophila germline stem cells. Dev Biol 2005. 